\title{
Expérience personnelle et expérience professionnelle dans l'enseignement de l'EPS : deux études de cas contrastées en didactique clinique
}

Pablo Buznic-Bourgeacq, André Terrisse et Ghislaine Lestel

\section{(2) OpenEdition}

\section{Journals}

Édition électronique

URL : https://journals.openedition.org/educationdidactique/366

DOI : 10.4000/educationdidactique.366

ISBN : 978-2-7535-1619-9

ISSN : 2111-4838

Éditeur

Presses universitaires de Rennes

\section{Édition imprimée}

Date de publication : 1 décembre 2008

Pagination : 77-95

ISBN : 978-2-7535-0790-6

ISSN : 1956-3485

\section{Référence électronique}

Pablo Buznic-Bourgeacq, André Terrisse et Ghislaine Lestel, « Expérience personnelle et expérience professionnelle dans l'enseignement de l'EPS : deux études de cas contrastées en didactique clinique ", Éducation et didactique [En ligne], 2-3 | Décembre 2008, mis en ligne le 01 décembre 2010, consulté le 16 août 2022. URL : http://journals.openedition.org/educationdidactique/366 ; DOI https://doi.org/10.4000/educationdidactique.366 


\title{
EXPÉRIENCE PERSONNELLE ET EXPÉRIENCE PROFESSIONNELLE DANS L'ENSEIGNEMENT DE L'EPS: \\ deux études de cas contrastées en didactique clinique
}

\author{
Pablo Buznic-Bourgeacq, André Terisse E Ghislaine Lestel, \\ DiDist, CREFI-T, IUFM Midi-Pyrénées, Université Toulouse 3
}

\begin{abstract}
Résumé : Cet article rend compte d'une articulation dialectique entre deux études de cas (Buznic-Bourgeacq, 2004 ; Lestel, 2005) centrées sur le même objet : le poids de l'expérience personnelle de l'enseignant dans une APSA (Activité Physique, Sportive et Artistique) sur son enseignement effectif. L'étude s'appuie sur le croisement de deux cas contrastés : E, une professeur d'EPS enseignant en milieu scolaire depuis 25 ans et ayant à enseigner, durant la recherche, un cycle de badminton, activité qu'elle pratique depuis plusieurs années, et un cycle de lutte, activité qu'elle n'a jamais pratiquée, et $\mathrm{D}$, professeur stagiaire durant sa première année d'enseignement en milieu scolaire et ayant à enseigner la danse, qu'elle pratique depuis longtemps et la lutte qu'elle n'a jamais pratiquée. Ainsi, on s'attache ici à examiner dans quelle mesure ces expériences spécifiques, personnelles et professionnelles, orientent la forme du processus de transmission des savoirs. Toutefois, notre ancrage épistémologique, la didactique clinique (Terrisse, 1994), nous conduit plus spécifiquement à identifier comment ces expériences singulières, constituées tout au long d'un processus de construction subjective, placent spécifiquement chaque sujet enseignant dans le système didactique et guident alors son enseignement. Ainsi, à partir de cadres d'analyse du didactique et du sujet enseignant, issus des didactiques disciplinaires et de la didactique clinique, inspirée de la clinique lacanienne, on tentera de montrer combien ces expériences, principalement l'expérience personnelle dans l'APSA, déterminent les enjeux subjectifs de l'enseignant inscrit dans le didactique et par conséquent le déroulement $\mathrm{du}$ processus didactique.
\end{abstract}

Mots-clés : didactique clinique de l'EPS, expériences personnelle et professionnelle, sujet enseignant

\section{Pablo Buznic-Bourgeacq, André Terrisse E Ghislaine Lestel}

\section{La problématique de l'expérience en didactique clinique de l'EPS}

Les pratiques d'enseignement en EPS (Éducation Physique et Sportive) constituent aujourd'hui un objet de recherche à part entière. Sous l'influence des sciences de l'éducation (Bru, 2004; Altet, 2002; Blanchard-Laville, Fablet, 2000) et des didactiques disciplinaires (Venturini, Amade-Escot, Terrisse, 2002; Sensevy, 2002), nombre de chercheurs en EPS ont orienté leur regard vers ces pratiques afin de tenter de les décrire, les comprendre et les expliquer. Renouant avec la perspective anthropologique en sciences humaines (Chevallard, 1991), ces recherches se sont intéressées à l'effectivité des pratiques professionnelles des sujets enseignants qu'elles observent et dont elles rendent compte. Souvent ancrée dans des IUFM (Institut Universitaire de Formation des Maîtres), l'intention de recherche qui se dessine est d'extraire de ces pratiques des indices de compétence professionnelle, d'expertise et d'expérience, d'adaptation personnelle afin de les caractériser, les systéma- tiser et éventuellement les utiliser après-coup dans des plans de formation des enseignants.

En ce qui nous concerne, dans ce vaste champ de l'analyse des pratiques d'enseignement en EPS, notre attention spécifique porte sur les expériences personnelle et professionnelle des enseignants d'EPS. Considérant qu'une expérience personnelle de pratiquant dans une APSA (Activité Physique, Sportive et Artistique) détermine en grande partie le contenu et la forme de son enseignement effectif, de même que l'expérience professionnelle accumulée par la pratique répétée de l'enseignement en milieu scolaire, nous nous sommes interrogés sur le poids de ces expériences dans l'enseignement de l'EPS. Nous rendons compte alors dans le cadre de cet article d'une articulation dialectique entre deux études de cas (BuznicBourgeacq, 2004; Lestel, 2005) centrées sur le même objet: le poids de l'expérience personnelle de l'enseignant dans une APSA sur son enseignement effectif. Mais, si cette articulation est justifiée par l'utilisation de cadres conceptuel et méthodologique iden- 
tiques, elle trouve particulièrement son intérêt par le fait que ces recherches ont été menées avec deux enseignantes ayant des expériences professionnelles bien différentes. L'une (D) est enseignante stagiaire (PLC2, Professeur des Lycées et Collèges $2^{\text {ème }}$ année) et se confronte pour la première fois à l'enseignement en milieu scolaire, l'autre (E) est professionnellement expérimentée puisqu'elle enseigne l'EPS depuis plus de vingt-cinq ans. Notre regard porte alors sur le croisement de ces expériences à partir d'un point commun: elles enseignent toutes les deux pour la première fois une APSA qu'elles n'ont jamais pratiquée, la lutte.

\begin{tabular}{|c|c|c|}
\cline { 2 - 3 } \multicolumn{1}{c|}{} & $\begin{array}{c}\text { Enseignante } \\
\text { professionnellement } \\
\text { débutante (D) }\end{array}$ & $\begin{array}{c}\text { Enseignante } \\
\text { professionnellement } \\
\text { expérimentée (E) }\end{array}$ \\
\hline $\begin{array}{c}\text { Expérience } \\
\text { personnelle }\end{array}$ & Danse & Badminton \\
\hline $\begin{array}{c}\text { Pas } \\
\text { d'expérience } \\
\text { personnelle }\end{array}$ & Lutte & Lutte \\
\hline
\end{tabular}

Tableau 1 : Expériences personnelle et professionnelle chez D et $\mathrm{E}$

Il s'agit alors ici d'examiner dans quelle mesure ces expériences spécifiques orientent la forme du processus de transmission des savoirs. Mais notre regard sur l'expérience et son poids sur l'enseignement effectif comporte une spécificité tenant à notre posture épistémologique. En effet, notre intention n'est pas véritablement d'extraire et de constituer des indicateurs d'une compétence professionnelle capitalisée par le biais de ces expériences. Il ne s'agit pas d'identifier, au travers d'une diversité d'items, les composants d'une expertise professionnelle (Tochon, 1996; Durand, 1998), mais bien de comprendre comment des expériences singulières, constituées tout au long d'un processus de construction subjective, placent spécifiquement chaque sujet enseignant dans le système didactique et guident alors son enseignement. Notre ancrage épistémologique en didactique clinique (Terrisse, 1994; Terrisse, 2005) oriente notre regard sur le didactique (Chevallard, 1991) et nous amène, dans notre démarche scientifique, à prendre en compte les sujets que l'on observe, leur histoire personnelle, leur « déjà-là » et les enjeux subjectifs qu'entraîne leur inscription dans un système didactique. On considère en effet que seule cette prise en compte permet au chercheur de donner du sens à leur expérience et de comprendre son poids sur la transmission du savoir.

Cadre conceptuel : logique du système didactique et logique du sujet enseignant

Dans l'optique de décrire à la fois le didactique et les sujets qui le constitue, la didactique clinique propose un système théorique spécifique, en empruntant aux cadres conceptuels des didactiques disciplinaires et de la didactique comparée, ainsi que de la psychanalyse, principalement de la clinique lacanienne. L'originalité de la démarche réside dans l'articulation entre des descripteurs du didactique, des descripteurs du sujet enseignant et un système interprétatif prenant à la fois en compte les contraintes didactiques et les contraintes internes au sujet enseignant. L'objectif de cette démarche en construction est de constituer une théorie du sujet didactique, c'est-à-dire un système théorique capable de proposer une modélisation du réseau de contraintes qui anime le sujet enseignant pris dans le didactique. On présente donc les différents descripteurs, appartenant aux deux catégories citées, pour ensuite tenter de les articuler au travers de notre système interprétatif spécifique.

\section{Une problématique transpositive}

Notre investigation menée sur l'expérience en didactique situe notre travail dans une problématique transpositive (Verret, 1975; Chevallard, 1991). En effet, notre intention est bien d'analyser comment une référence extérieure au système didactique se trouve transposée en objets de savoirs enseignés à des élèves dans l'institution scolaire. Toutefois, la question de la référence (Terrisse, 2001) ne renvoie pas ici à proprement parler à un « savoir savant » (Chevallard, 1991) ou à « une pratique sociale de référence » (Martinand, 1994). La légitimité externe des objets de savoir qui pénètrent l'espace didactique de la classe est ici prise en compte à partir du sujet enseignant, dont l'expérience personnelle constitue alors la référence. Il nous semble en effet que dans l'institution classe, les savoirs considérés comme définitivement légitimes pour les élèves sont ceux qui appartiennent en dernier ressort au sujet enseignant. Nous décrivons ainsi le processus de transpo- 
sition des savoirs à partir de ce sujet qui le génère et l'oriente et le définissons en terme de « conversion didactique " (Buznic-Bourgeacq, 2005) : la conversion d'une expérience subjective en objets de savoirs enseignés. L'intérêt d'une telle distinction repose alors sur deux points. D'une part, elle nous permet de questionner les savoirs enseignés au-delà d'une référence institutionnelle, en prenant en compte la singularité de l'expérience qui la soutient. D'autre part, elle nous incite à identifier comment cette référence expérientielle, lorsqu'elle s'inscrit dans une intention didactique, oriente, en parallèle le fonctionnement du système didactique lui-même.

\section{Des outils pour décrire le didactique}

Pour comprendre le poids de cette expérience, il s'agit en premier lieu d'expliciter les différents aspects du fonctionnement didactique sur lesquels notre regard sera posé. Trois points sont ainsi au centre de nos préoccupations.

\section{La dialectique planification/mise en œuvre}

Toute pratique enseignante est fondée sur une planification. Elle peut être plus ou moins explicite, plus ou moins structurée et hiérarchisée, plus ou moins précise, mais toute pratique didactique repose sur des intentions didactiques appartenant en grande partie à l'enseignant (Brousseau, 1986). Chaque enseignant, avant d'entrer dans la classe, possède des objectifs didactiques, sait dans une certaine mesure quels objets de savoir il compte enseigner et quelles situations il mettra en place pour soutenir cette transmission. Mais toute pratique effective ne renvoie évidemment pas à la simple mise en oeuvre de cette planification, même si elle est particulièrement précise et structurée pour certains enseignements. La contingence du processus de transmission, due principalement à l'incertitude des interactions, est inévitable (Brousseau, 1998; Terrisse, 1994). Ce que l'enseignant compte transmettre aux élèves et ce qu'il leur transmet effectivement en classe est rarement identique. Ainsi, l'analyse des écarts entre la prévision d'objets de savoirs à transmettre et leur mise en ouvre effective constitue un des outils d'analyse les plus utiles de la recherche en didactique. Ces écarts ont été étudiés sous diverses facettes et ont été théorisés en EPS en terme de différence entre inten- tions et décisions (Carnus, 2002), en mathématiques puis dans de nombreuses disciplines en terme de distinction entre savoirs à enseigner et savoirs enseignés (Chevallard, 1991), au point de constituer un des principaux fondements d'une méthodologie de recherche, l'ingénierie didactique (Artigue, 1990). Ainsi, notre intention est ici d'examiner dans quelle mesure les expériences personnelle et professionnelle qui servent de référence à nos deux enseignantes dans leurs prévisions didactiques, leur permettent alors de faire face à la contingence du processus didactique.

La dialectique dévolution/institutionnalisation

Les deux concepts formant cette dialectique ont été élaborés en didactique des mathématiques par Brousseau (1986). Rejetant les pratiques d'enseignement fondées sur la communication magistrale et injonctive d'objets de savoir, il a voulu fonder celles-ci sur la dévolution de problèmes aux élèves, condition, pour lui, nécessaire à leur apprentissage. Ainsi conçue, la dévolution renvoie à l'activité de l'enseignant consistant à mettre en place des situations dans lesquelles la responsabilité de l'émergence du savoir est déléguée aux élèves par l'intermédiaire de leur activité adaptative. Puisque l'enseignant ne peut pas apprendre à la place de ses élèves, il a le devoir de créer un milieu favorable à cette émergence. Dans cette perspective, de par leur activité, les élèves ont alors à construire leurs propres savoirs. Mais, cette dévolution n'est qu'un déguisement didactique de l'intention du professeur, puisque celui-ci tente de faire construire aux élèves un savoir qui est en fait déjà historiquement constitué et reconnu, qui fait partie du capital épistémologique et culturel du champ d'activité qu'il enseigne (Brun, 1996). Si l'enseignant doit laisser les élèves construire par eux-mêmes leurs propres savoirs, il doit bien aussi à un moment donné, réinscrire ces savoirs dans l'univers symbolique des savoirs reconnus et partagés. Il doit reconnaître et s'entendre avec toute la classe sur ce qu'il faut retenir et conserver. L'un de ses rôles consiste alors à figer symboliquement les « bons savoirs » (Chevallard, 1989) en les faisant entrer dans un processus d'institutionnalisation (Brousseau, 1986). Ainsi, le rôle de l'enseignant vis-à-vis des savoirs est à double face: il «souhaite que l'élève veuille ne tenir la réponse que de lui-même mais, en même temps, il veut, il a le devoir social que l'élève donne la bonne réponse » (Brousseau, 1998). Cette dialectique, qui a dans une 
certaine mesure justifiée l'émergence de la recherche en didactique (Brun, 1996), est alors au centre de nos préoccupations. Il s'agit pour nous d'identifier dans quelle mesure les expériences personnelle et professionnelle des enseignantes observées les conduisent à utiliser, valoriser ou minorer ces deux processus. On examinera ainsi, au regard de ces expériences, comment les phases de dévolution et d'institutionnalisation s'articulent et dans quelle mesure elles leur semblent utiles, nécessaires voire incontournables.

\section{L'ostension des savoirs}

Lorsqu'un problème a été dévolué aux élèves, il est rare que la simple activité adaptative des élèves conduise directement à l'émergence d'un savoir. D'une part, le principal obstacle sous-jacent à cette adaptation est souvent difficilement surmonté à court terme. D'autre part, la complexité des pratiques motrices sous-tend, au-delà du problème majeur posé aux élèves, une multiplicité d'obstacles difficiles à prévoir dans toute leur diversité. Ainsi, un des rôles de l'enseignant consiste à gérer comme il le peut cette diversité d'obstacles. En effet, de sa nécessité de maintenir la relation didactique avec ses élèves (Sensevy, Mercier, Schubauer-Léoni, 2000), il ne peut les laisser dans une situation d'échec permanente. Alors, au travers d'interactions didactiques ponctuelles, il peut orienter ses interventions à partir des différents savoirs sous-jacents aux multiples obstacles rencontrés par les élèves, en délaissant certains et en valorisant d'autres, en s'attachant à l'obstacle principal ou en intervenant à propos de tous ceux qui émergent de manière erratique et imprévisible dans la classe. Mais, il peut aussi intervenir de différentes manières. Face aux obstacles des élèves, il ne peut éviter de reprendre à son compte certaines responsabilités vis-à-vis de l'émergence des savoirs. Il peut alors « donner à voir » du savoir au travers de procédures ostensives (Salin, 2002). Nous avons ainsi distingué trois principales formes d'ostensions, les plus récurrentes en EPS: lorsque l'enseignant explicite par le discours des éléments structurels ou fonctionnels du savoir, lorsqu'il contraint la motricité de l'élève en manipulant son corps, ou lorsqu'il démontre le savoir dans sa globalité par l'intermédiaire de son propre corps. Notre intérêt consiste alors ici à examiner dans quelle mesure les différentes expériences des enseignantes observées les amènent, voire les contraignent, à utiliser spécifiquement ces différentes formes et à orienter leurs interventions ostensives vers des obstacles spécifiques, pour atteindre les apprentissages visés par l'enseignement.

Des outils pour décrire et interpréter la logique subjective de l'enseignant

Pour comprendre les ressorts de l'expérience du sujet enseignant, il faut aussi pouvoir la décrire et pour comprendre son impact sur les processus didactiques, il faut un système d'interprétation spécifique. Notre option épistémologique en didactique clinique nous amène à prendre en compte cette expérience non pas comme une simple variable, mais bien comme un déterminant qu'il s'agit de théoriser de manière qualitative pour en montrer l'efficience.

$$
\text { Le « déjà-là » du sujet enseignant }
$$

Les premières ingénieries menées en mathématiques dans les années soixante-dix ont très vite été confrontées à un obstacle: la singularité du rapport de l'enseignant aux savoirs à enseigner. En effet, lorsque le didacticien tente d'identifier l'appropriation d'un contenu spécifique par les élèves, il se heurte en premier lieu à la transmission personnelle qu'en fait le professeur. Ainsi, les didacticiens ont rapidement dû tenter d'identifier cette singularité de l'enseignant face aux savoirs à enseigner. On trouve alors dans les théories didactiques, voire par extension en sciences de l'éducation, plusieurs concepts qui lui donnent une voie de théorisation. «Épistémologie professionnelle » (Brousseau, 1998), «idéologie épistémologique » (Perrin-Glorian, 1994), « conceptions » (Giordan, 1996), « rapport au (x) savoir(s)» (Beillerot, 1989; Chevallard, 1989; Charlot, 1997), sont autant d'apports théoriques permettant de décrire différents aspects de la position spécifique du sujet enseignant face aux savoirs. Il y a du « déjà-là » (Terrisse, 2001) chez l'enseignant lorsqu'il rentre dans la classe. Il s'est déjà confronté, la plupart du temps, aux savoirs qu'il va enseigner et il a une conception personnelle de ces savoirs, de leur articulation, voire de leur transmission. Le poids de ce « déjà-là » sur la transmission effective des savoirs est alors fondamental et la problématique des expériences personnelle et professionnelle y devient centrale (Loizon, 2004). Ainsi, nous avons pris en compte ce « déjà-là » sous trois aspects nous permet- 
tant de décrire la singularité de l'enseignant face aux APSA qu'il doit enseigner:

- le rapport à l'expérience personnelle dans l'APSA: il s'agit là d'identifier les lieux, les institutions et les temporalités de sa pratique personnelle dans chaque APSA, ainsi que les affects associés à cette pratique ou à l'absence de pratique.

- la conception de l'APSA : il s'agit d'examiner la manière dont il formalise chaque APSA, sa logique interne, ses principaux savoirs, leur articulation, leur hiérarchisation.

- la conception de l'enseignement de l'APSA: il s'agit de caractériser, dans la continuité de la conception de chaque APSA, les savoirs qu'il considère comme fondamentaux à transmettre ou à éviter de transmettre.

Ces trois analyseurs, s'ils restent qualitatifs, nous permettront de comparer les « déjà-là » de chaque enseignant au regard de leur expérience personnelle. Puis, ils nous permettront d'établir une première comparaison entre nos deux enseignantes, avant même de confronter leurs pratiques, et ainsi d'identifier les premiers indicateurs d'une expérience professionnelle.

Les enjeux subjectifs de l'enseignant dans le didactique

Les indicateurs que nous venons de présenter nous permettent de décrire le processus de transmission ainsi que l'expérience qui soutient celui ou celle qui le met en ouvre. Mais notre intention reste de caractériser le jeu de déterminations qui s'instaure entre cette expérience et ce processus. Comme nous l'avons précisé, notre posture épistémologique spécifie notre regard sur ce jeu de déterminations. En effet, pour interpréter les processus didactiques au regard des expériences des enseignantes, on considère qu'il faut tenir compte des contraintes inhérentes au système didactique, en intégrant les « contraintes internes » qui pèsent sur le sujet enseignant, c'est-à-dire « les contraintes que le sujetprofesseur s'impose à lui-même, ou plutôt que sa part inconsciente lui impose, à son insu même » (Blanchard-Laville, 2001). Car, inscrit dans le didactique, la place de l'enseignant le soumet à divers enjeux qui dépassent les enjeux proprement didactiques: il a une position symbolique à assumer, une image de lui-même à exposer aux élèves et une réelle contingence à affronter dans la conduite de sa classe. Ainsi, nous identifierons le jeu de déterminations qui s'instaure entre les expériences des enseignantes et les processus didactiques précédemment décrits à partir de trois cadres d'analyse:

- le rapport de l'enseignant à la contingence: toute situation d'enseignement est contingente. Il est impossible pour l'enseignant de prévoir tous les obstacles des élèves et les demandes qui en découleront. Ainsi, lorsqu'il rentre dans la classe, il sait qu'une part de maîtrise lui échappera inévitablement, qu'il devra se confronter au réel de la classe et s'en « arranger» comme il le peut. Nous postulons alors que son « déjà-là » sera sa principale ressource pour réintégrer sa part de maîtrise didactique de la classe. Ainsi, la spécificité de ses expériences personnelle et professionnelle le marquera d'un rapport spécifique à la contingence du processus de transmission, un rapport allant du plus craintif au plus « jubilatoire». Ainsi, les stratégies didactiques qu'il mettra en œuvre pour maîtriser cette contingence seront les révélateurs de ce rapport spécifique.

- le «sujet supposé savoir»: ce concept a été élaboré en psychanalyse par Lacan (1966) pour caractériser la position symbolique de l'analyste, place vers laquelle le transfert de l'analysant se dirige. Il a été transposé en didactique par Chevallard (1991), ou encore Blanchard-Laville (2001), pour situer le sujet enseignant dans sa position de sujet sachant face aux élèves. De ce fait, il nous semble que la recherche et le maintien d'une telle position constituent un arrière-plan permanent de l'activité enseignante, puisqu'elle constitue le fondement de sa légitimité didactique et donc professionnelle. Les expériences personnelle et professionnelle du sujet enseignant sont un point d'appui fondamental à cette légitimité. Ainsi, cet enjeu symbolique sous-jacent et constitutif de l'activité didactique de l'enseignant ne peut pas être esquivé par celui qui veut comprendre, voire expliquer le fonctionnement du processus de transmission.

- l'impossible à supporter: ce syntagme est une des définitions du réel, tel qu'il est proposé dans la topologie lacanienne de la structure du sujet. Puisque 
l'enseignant ne peut pas tout maîtriser d'un point de vue didactique dans le réel de la classe, il doit s'attacher à faire au mieux pour chacun des élèves. Mais, son « déjà-là » oriente ses choix didactiques in situ, dans sa capacité à gérer le temps didactique (Mercier, 1999), l'hétérogénéité des élèves, la diversité des enjeux didactiques. On postule alors que ce « déjà-là », actualisé dans le réel de la classe, oriente ses choix à son insu, le rendant incapable de supporter certains aspects du fonctionnement didactique des séances d'enseignement pour des raisons qui lui sont propres et dont il n'a pas toujours conscience.

L'articulation de ces trois cadres d'analyse nous sert ainsi à proposer une base interprétative du rapport entre les expériences des enseignantes et les phénomènes didactiques observés.

\section{Méthodologie: une comparaison en didactique clinique}

\section{Le recueil des données}

Pour pouvoir décrire et comparer les expériences singulières et les différents enseignements menés par chacune des enseignantes à partir des axes théoriques que nous venons de proposer, nous avons mené des recueils de données identiques pour les deux cas. La recherche a été menée pour chaque enseignante à partir de l'analyse de deux séances d'enseignement de chaque APSA, conduites les mêmes semaines avec la même classe. Les recueils de données s'organisent alors en cinq temps respectifs :

- des entretiens semi-directifs préalables, dits expérientiels, consistant à repérer le « déjà-là » des enseignantes dans chaque APSA, effectués avant la conduite des cycles observés

- des analyses des fiches de préparation de séances, consistant à identifier les différents objets de savoirs qu'elles avaient prévu de transmettre pour chaque séance de chaque APSA,

- des observations vidéos in situ, consistant à examiner les objets de savoir effectivement transmis, l'importance accordée aux phases de dévolution et d'institutionnalisation, ainsi que les différentes formes de procédures ostensives utilisées,

- des entretiens post-séances, consistant à reconnaître les objets de savoirs effectivement transmis par les enseignantes,

- des entretiens « d'après-coup » (Terrisse, 2000), effectués plusieurs mois après les observations, consistant à constater le point de vue interne des sujets sur leur pratique effective.

\section{L'analyse didactique comparative}

À partir de ces données recueillies et de nos outils théoriques, nous avons établi des comparaisons sur les mêmes indicateurs entre chacun des enseignements menés.

En ce qui concerne la dialectique planification/ mise en œuvre, Nous avons examiné si les savoirs transmis ont été prévus par l'enseignante ou s'ils émergent dans le vif des séances, en réponse à l'activité maladroite ou inadaptée des élèves. Nous avons alors regardé dans quelle mesure chaque enseignante a tenté de répondre à la diversité des obstacles rencontrés par les élèves ou s'est attachée à suivre le fil de l'avancée du savoir tel qu'elle l'avait prévu.

Ensuite, à propos de la dialectique dévolution/ institutionnalisation, nous avons examiné si chaque savoir ayant émergé dans la classe a été le produit de l'activité adaptative des élèves confrontés à un milieu problématique ou si l'enseignante a endossé la responsabilité de son émergence à part entière. Il s'est agi d'analysé la récurrence des phases d'institutionnalisation et on a identifié pour chaque savoir transmis par l'enseignante dans une interaction ponctuelle s'il avait été préalablement institutionnalisé.

Enfin, vis-à-vis de la problématique de l'ostension, nous avons examiné pour l'ensemble des savoirs mis en jeu par l'enseignante dans ses interactions, à partir de quelle forme d'ostension, telles que nous les avons catégorisées précédemment, elle mettait en jeu le savoir.

Il ne nous restait alors plus qu'à réinscrire le sujet au centre de ces analyses. 
Une démarche clinique d'interprétation

Au-delà de notre système théorique qui propose une modélisation du sujet pris dans le didactique, notre posture clinique nous amène à prendre en compte la subjectivité des enseignantes d'un point de vue méthodologique. Cette prise en compte est alors effectuée à deux niveaux:

- d'une part, il s'agit d'identifier les « déjà-là » des enseignantes afin de rendre compte de leurs expériences subjectives respectives. Ainsi, au travers des entretiens préalables, on a tenté d'avoir accès à leurs expériences, non pas pour en faire de simples variables auxquelles on attribuerait les spécificités des processus didactiques, mais bien pour les décrire qualitativement et identifier dans leur singularité des éléments déterminants de ces spécificités dans leur enseignement. À partir des trois aspects du « déjàlà » précédemment décrits, on a identifié la diversité des objets de savoir constitutifs de leurs expériences, leur inscription dans l'histoire institutionnelle et affective des enseignantes et dans les conceptions épistémologique et didactique qu'elles ont des différentes APSA enseignées.

- d'autre part, si l'identification de ces « déjà-là » permet d'examiner qualitativement ce qui dans les expériences personnelle et professionnelle des enseignantes semble déterminer le contenu et la forme du processus effectif de transmission des savoirs, cette interprétation reste du point de vue du chercheur. Alors, pour comprendre ce jeu de déterminations dans une démarche clinique, il s'agit aussi de l'identifier du point de vue du sujet enseignant. Ainsi, les entretiens d'après-coup nous permettent d'interroger les enseignantes sur leur ressenti différentiel dans chaque enseignement mené et sur les causes perçues des processus didactiques spécifiques préalablement décrits par le chercheur. La validation d'une telle démarche méthodologique consiste alors dans la rencontre d'une subjectivité identifiée de manière externe par le chercheur, corrélée à des descriptions des pratiques effectives avec sa reconnaissance interne et son interprétation par le sujet lui-même (Van Der Maren, 1995).

\section{Résultats: deux enseignantes, quatre expériences, quatre enseignements}

\section{Les « déjà-là » des enseignantes $\mathrm{D}$ et $\mathrm{E}$ :}

Pour une facilité de lecture, nous proposons l'analyse des « déjà-là » sous la forme du tableau comparatif suivant, effectué à partir des données recueillies lors des entretiens "expérientiels » et regroupées et organisées selon les axes présentés dans le cadre théorique. 


\section{EXPÉRIENCE PERSONNELLE ET EXPÉRIENCE PROFESSIONNELLE DANS L'ENSEIGNEMENT DE L'EPS...}

\section{Buznic-Bourgeacq \& al}

\begin{tabular}{|c|c|c|c|}
\hline & & Enseignante professionnellement débutante (D) & $\begin{array}{l}\text { Enseignante professionnellement } \\
\text { expérimentée (E) }\end{array}$ \\
\hline \multirow{3}{*}{ 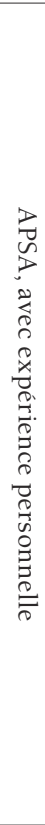 } & $\begin{array}{l}\text { Rapport à l'expé } \\
\text { perso dans l'APSA } \\
\text { dont elle est } \\
\text { spécialiste }\end{array}$ & $\begin{array}{l}\text { - Une pratique longue et clivée: "J'ai fait de la } \\
\text { danse classique, de six à treize ans et de seize à } \\
\text { dix-huit ans »; "j'ai fait du contemporain (option } \\
\text { CAPEPS), quatre heures de danse au moins par } \\
\text { semaine plus autant en théorie " } \\
\text { - Un clivage fondamental: " (la contemporaine) } \\
\text { a beaucoup plus de sens qu'une chorégraphie } \\
\text { carrée... c'est une chorégraphie où ça sort de toi »; } \\
\text { "mais il y a des fois, ça ne ressemble plus trop à } \\
\text { de la danse » }\end{array}$ & $\begin{array}{l}\text { - Une pratique personnelle: "J'ai un vécu } \\
\text { dans les activités de raquettes, dans le } \\
\text { squash". } \\
\text { - Un engagement dans le sport scolaire: Je le } \\
\text { (le badminton) fais régulièrement "pratiquer } \\
\text { en UNSS depuis au moins six ans". }\end{array}$ \\
\hline & $\begin{array}{l}\text { Conception de } \\
\text { l'APSA }\end{array}$ & $\begin{array}{l}\text { - Une grande diversité de savoirs articulés et } \\
\text { hiérarchisés: à propos du «temps », de « l'espace ", } \\
\text { de "l'énergie », de " la relation aux autres », des } \\
\text { " différents rôles "... } \\
\text { - Une structure de l'APSA divisée en deux principaux } \\
\text { aspects: "la rigueur technique et esthétique » de } \\
\text { la danse classique et « l'interprétation expressive » } \\
\text { de la contemporaine }\end{array}$ & $\begin{array}{l}\text { - Une activité d'opposition: « c'est une } \\
\text { activité duelle, d'opposition. Tu es en face } \\
\text { d'un volant, d'un adversaire dont on va } \\
\text { essayer de voir comment il joue ». } \\
\text { - Une activité d'analyse du rapport de force: } \\
\text { "trouver ses points forts et ses points faibles } \\
\text { pour gagner» }\end{array}$ \\
\hline & $\begin{array}{l}\text { Conception de } \\
\text { l'enseignement de } \\
\text { l'APSA }\end{array}$ & $\begin{array}{l}\text { - Intention d'enseigner tous les savoirs énumérés } \\
\text { précédemment } \\
\text { - Éviter de transmettre « des gestes, des formes, ou } \\
\text { des techniques décontextualisés ", afin de «faire } \\
\text { vivre une chorégraphie avant tout » }\end{array}$ & $\begin{array}{l}\text { - Intention d'enseigner les éléments } \\
\text { stratégiques de cette activité d'opposition.: } \\
\text { "l'analyse du jeu de l'adversaire » à partir } \\
\text { d'une organisation en " poule de niveaux» }\end{array}$ \\
\hline \multirow{3}{*}{ 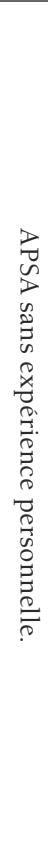 } & $\begin{array}{l}\text { Rapport à l'expé } \\
\text { perso dans l'APSA } \\
\text { dont elle n'est pas } \\
\text { spécialiste }\end{array}$ & $\begin{array}{l}\text { - Une pratique inexistante en lutte, une brève } \\
\text { expérience en judo: «j'ai jamais fait de lutte »; } \\
\text { "j’ai fait du judo en STAPS, en première année » } \\
\text { - Un rejet de l'APSA lié à des apprentissages } \\
\text { douloureux et dénués de sens: «J'ai détesté »; } \\
\text { «j'avais beaucoup de blessures "; «j'aimais pas } \\
\text { ça, c'était de l'apprentissage de gestes »; "ça ne } \\
\text { me plaisait pas, parce qu'il n'y a aucune logique } \\
\text { par rapport au sens de l'activité " }\end{array}$ & $\begin{array}{l}\text { - Une ancienne pratique de judo «J'ai un } \\
\text { vécu très ancien, vers l'âge de quatorze ans, } \\
\text { de judo ». } \\
\text { - Une grande distance avec l'activité combat: } \\
\text { "Ce vécu-là, il est très loin ». } \\
\text { - Au niveau de la formation initiale: " après } \\
\text { rien au niveau de la formation UFRSTAPS, } \\
\text { en combat ». } \\
\text { - Mais un stage en lutte en formation } \\
\text { continue: « je reporte l'activité lutte sur cette } \\
\text { classe-là ». }\end{array}$ \\
\hline & $\begin{array}{l}\text { Conception de } \\
\text { l'APSA }\end{array}$ & $\begin{array}{l}\text { - Une confusion entre judo et lutte: prise en compte } \\
\text { des techniques d'immobilisation, normalement } \\
\text { exclusives au judo } \\
\text { - Deux principaux types de savoirs: sécuritaires } \\
\text { (« savoir chuter et faire chuter en toute sécurité ») } \\
\text { et tactique/stratégiques ("utiliser la force de } \\
\text { l'autre», « le principe d'action/réaction») }\end{array}$ & $\begin{array}{l}\text { - Un traitement envisagé sur le mode } \\
\text { "affectif " d'opposition, "étant donné } \\
\text { que je n'ai pas une grande maîtrise (des) } \\
\text { compétences spécifiques ", "renforcer } \\
\text { la confiance en soi... pour que les élèves } \\
\text { maîtrisent le facteur émotionnel et pour } \\
\text { qu'ils s'adaptent à un rapport de force ». }\end{array}$ \\
\hline & $\begin{array}{l}\text { Conception de } \\
\text { l'enseignement de } \\
\text { l'APSA }\end{array}$ & $\begin{array}{l}\text { - Intention d'enseigner tous les savoirs énumérés } \\
\text { précédemment } \\
\text { - Éviter de transmettre des gestes décontextualisés: } \\
\text { "qu'ils soient dans une logique... pas de } \\
\text { reproduction de formes techniques mais de } \\
\text { combattre.» }\end{array}$ & $\begin{array}{l}\text { - Insistance des éléments techniques: } \\
\text { «développer les techniques spécifiques... } \\
\text { apprendre à chuter ou à faire chuter,... } \\
\text { enchaîner des attaques". } \\
\text { - }\end{array}$ \\
\hline
\end{tabular}

Tableau 2: Les déjà-là de D et E 
La dialectique planification/mise en ouvre chez D et $\mathrm{E}$

On présente ici quatre schémas représentant un aspect de la chronogenèse (Chevallard, 1991) des objets de savoir sur toute la longueur des premières séances de chaque enseignement observé. Les différentes séances de chaque cycle, d'une durée d'environ une heure et demie, étant particulièrement semblables vis-à-vis de nos objets de recherche, nous nous attachons, par souci de lisibilité, à décrire pour chaque enseignement la première d'entre elles. Nous identifions, pour chaque intervention des enseignantes auprès des élèves, les différents savoirs mis en jeu que l'on comptabilise au fur et à mesure de leur apparition pour l'ensemble de la séance. Ces savoirs sont codés de manière chiffrée et continue selon leur apparition progressive dans chaque séance (S1, S2, S3, Sn). Chaque solution apportée par les enseignantes aux obstacles, potentiels ou effectifs, des élèves est identifiée comme un savoir et chaque savoir est défini et catégorisé par le chercheur en référence aux objets de savoir identifiables dans les programmes du collège. Nous distinguons alors ici deux types de savoirs: ceux qu'elles avaient prévu d'enseigner, soit les enjeux didactiques des situations, tels qu'ils sont décrits dans les fiches de préparation, et ceux qu'elles font émerger in situ au regard de l'activité des élèves.

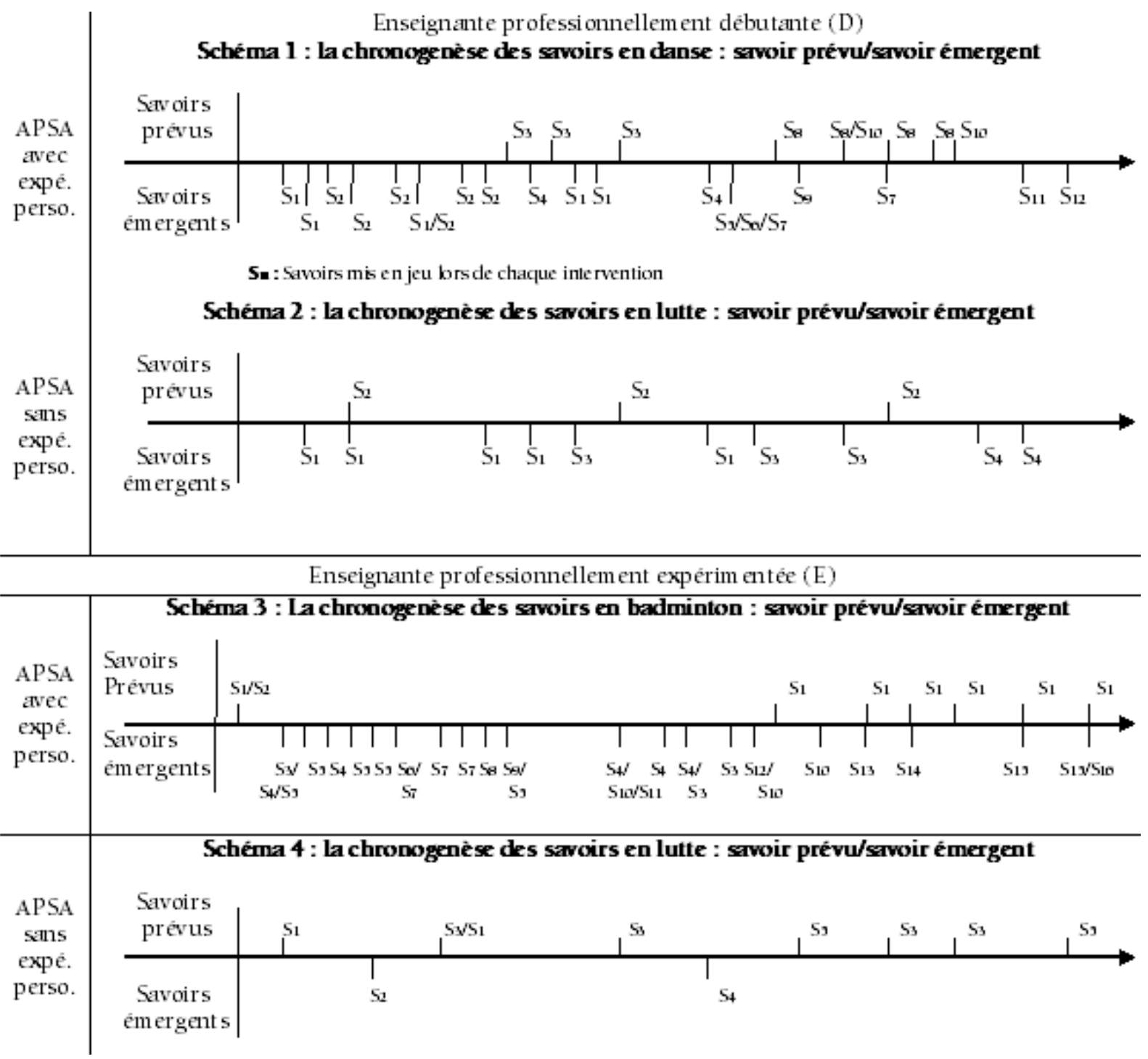

Tableau 3: la chronogenèse des savoirs: savoir prévu/savoir émergent 
Nous pouvons dès lors remarquer plusieurs similitudes et différences entre ces quatre séances, qui présentent des dynamiques singulières:

- chez D comme chez E, les enseignements s'appuyant sur une expérience personnelle de pratiquant sont scandés par une grande diversité d'interventions ( 23 pour D et 22 pour E), elles-mêmes appuyées par une multiplicité de savoirs ( 12 pour D et 16 pour E). À l'inverse, dans les enseignements sans expérience personnelle, peu de savoirs sont mis en jeu (4 pour D et 5 pour $\mathrm{E}$ ), au travers de quelques interventions (12 pour D et 9 pour E).

- chez D et E, dans les enseignements de leurs spécialités, la danse et le badminton, une grande majorité des savoirs transmis émerge in situ, en réponse aux obstacles des élèves, sans que leur transmission n'ait été prévue a priori.

D avait prévu de centrer son cours sur la gestion de l'espace scénique (S10) et sur la connaissance des éléments techniques de la danse (S3) et leur interprétation expressive (S8). Face à l'activité maladroite de plusieurs élèves, elle s'engage dans une multiplicité d'interactions didactiques ayant pour contenu une diversité de savoirs renvoyant à l'esthétique des postures et des mouvements (S1), à l'engagement émotionnel (S4) ou encore aux relations entre les danseurs (S14).

D'une manière similaire, E, qui avait prévu de centrer son cours de badminton sur la gestion de la position de l'adversaire (S1) et la maîtrise des trajectoires tendues (S2), répond aux divers obstacles des élèves à partir de nombreux savoirs relatifs à la maîtrise du dégagé (S5) et de l'amorti (S6), à l'efficacité des postures (S12) et mouvements offensifs (S10) ou encore au replacement défensif (S13).

Ainsi, en danse et en badminton, face à la contingence inhérente à l'activité d'apprentissage des élèves, les deux enseignantes s'autorisent de manière récurrente des écarts vis-à-vis des enjeux didactiques prévus des séances pour répondre à leurs obsta- cles singuliers. A fortiori chez D, l'enseignement mené consiste en une intervention incessante et multidirectionnelle.

- Par contre, chez D et E, les enseignements de lutte n'ont pas exactement la même forme. Si dans les deux enseignements, peu de savoirs émergent in situ sans que leur transmission n'ait été prévue, l'enseignement mené par E s'appuie sur une planification détaillée, ayant pour contenu plusieurs techniques efficaces de saisie (S1) et la gestion de la proximité (S3) et du maintien de la saisie (S5) et laissant peu de place à l'émergence de savoirs dans le vif des séances. En revanche, chez D, seul un savoir enseigné a été prévu, il renvoie à l'utilisation des forces de son adversaire pour le déséquilibrer (S2). Ainsi, dans le vif des séances, au regard des multiples obstacles des élèves, face auxquels le savoir S1 ne peut être utile, l'enseignante débutante met en jeu quelques savoirs non prévus renvoyant à l'utilisation du poids du corps (S1) et à la proximité (S3) et à la souplesse des saisies (S4). Il semble alors que si leur planification leur permet de faire face à la contingence du processus de transmission, D parvient tout de même quelque fois à prendre en compte la singularité des obstacles des élèves. Nous verrons, dans la partie suivante, que la forme spécifique de cette prise en compte relativise ce constat.

La dialectique dévolution/institutionnalisation chez $D$ et $\mathrm{E}$

Nous présentons ici quatre schémas représentant un autre aspect de la chronogenèse des savoirs pour les quatre séances déjà analysées. Nous y distinguons deux types d'interventions de la part des enseignantes: les interventions ponctuelles et les phases d'institutionnalisation. Nous précisons alors, pour chaque savoir mis en jeu, s'il a été institutionnalisé antérieurement ou s'il est mis en jeu sans avoir déjà été reconnu comme un enjeu officiel dans l'institution classe. Ainsi, chaque savoir mis en jeu par l'une des deux enseignantes peut soit avoir été déjà institutionnalisé auparavant (SnO: Savoir Officiel) soit ne jamais l'avoir été (Sn: Savoir). 


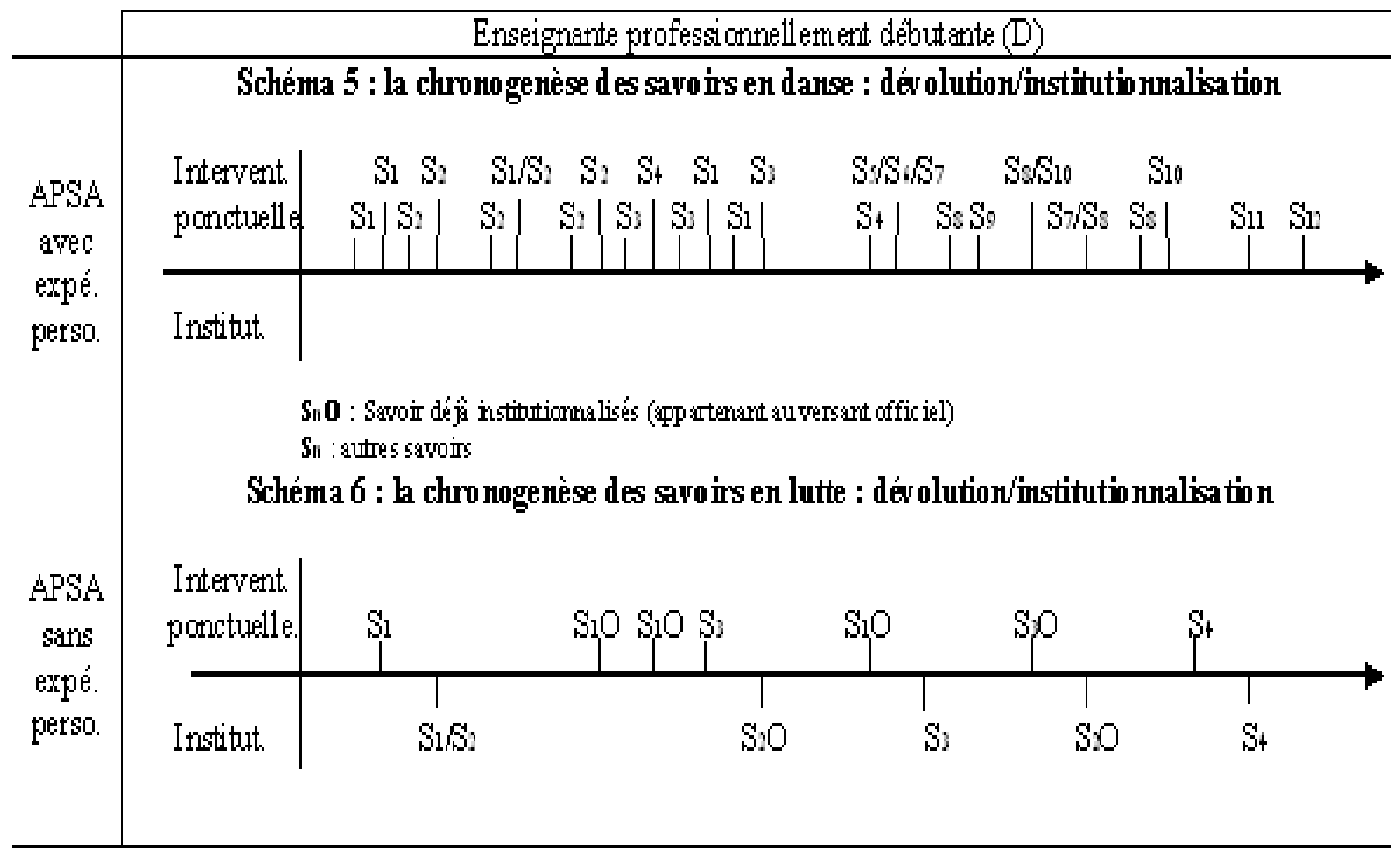

Enseignante professi onnell ement expérimentée $(\mathrm{E})$

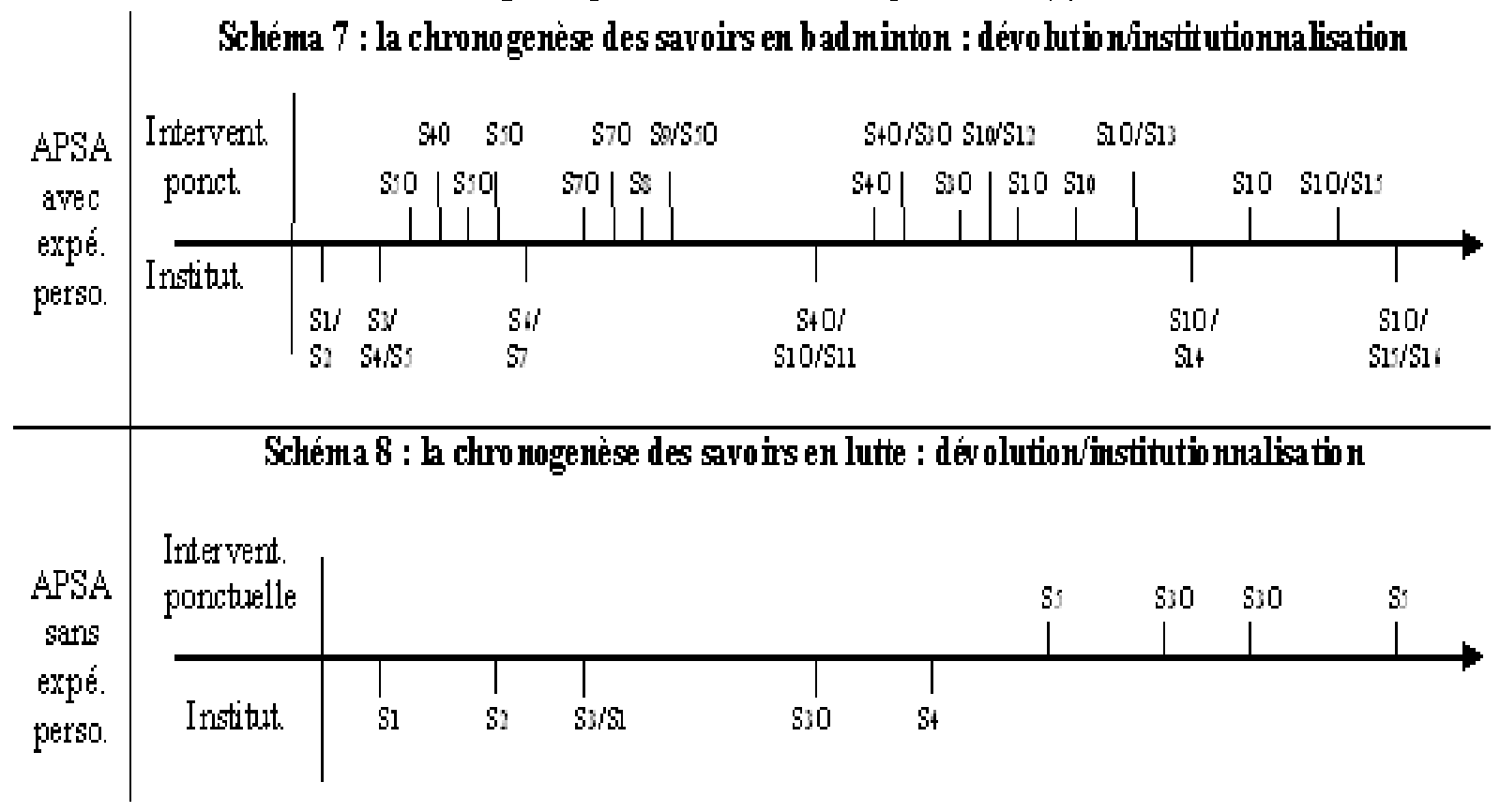

Tableau 4: la chronogenèse des savoirs: dévolution/ 


\section{Buznic-Bourgeacq \& al}

Plusieurs similitudes et différences apparaissent entre ces quatre dynamiques:

- Si les deux enseignantes dans chaque activité s'attachent à dévoluer des problèmes aux élèves pour qu'ils construisent leurs propres savoirs, les deux séances de lutte et celle de badminton sont ponctuées par de nombreuses phases d'institutionnalisation ( 5 pour D en lutte, 6 pour E en badminton et 5 pour E en lutte). Ces phases, dans la mesure où elles précèdent ou inaugurent les situations proposées aux élèves, remettent en question l'existence d'une véritable dévolution, puisque les solutions aux problèmes sont présentées à toute la classe par l'enseignante avant même que les élèves ne puissent se confronter aux milieux censés faire émerger ces solutions.

- De même, l'enseignement de danse mené par D est, dans cette analyse, très spécifique, puisqu'il ne comporte aucune phase d'institutionnalisation. L'enseignante, qui intervient inlassablement auprès des élèves, ne s'arrête jamais pour figer symboliquement un savoir pour l'institution classe. Aucun savoir ne prend alors le statut d'enjeu officiel.

- Dans les trois enseignements qui comportent des phases d'institutionnalisation, ceux de lutte et de badminton, l'articulation dans le temps entre le contenu des différentes interventions spécifie chacun d'entre eux. L'enseignement de badminton prend une forme relativement classique en milieu scolaire. E présente les consignes des situations à la classe, puis les élèves entrent en activité et $\mathrm{E}$ régule cette activité. Toutefois, la spécificité de son enseignement renvoie au fait que les phases d'institutionnalisation précèdent celles de dévolution, dans la mesure où la présentation des consignes est toujours accompagnée de la présentation à la classe des solutions aux problèmes dévolués.

- Pour autant, l'enseignement de lutte mené par E est, lui, plus original. En effet, les rares interventions de E sont majoritairement des phases d'institutionnalisation. La première moitié de son cours ne comporte aucune intervention qui ne s'adresse pas à toute la classe et aucun des savoirs émergents n'est mis en jeu de manière ponctuelle.

- Enfin, l'enseignement de lutte mené par D prend une forme très singulière. En effet, les phases d'insti- tutionnalisation sont presque toujours en continuité avec les interventions ponctuelles. Naviguant sur le tatami, lorsqu'elle parvient à trouver un moyen d'aider un élève dans son activité d'apprentissage, elle arrête la séance pour institutionnaliser le savoir mis en jeu dans l'intervention ponctuelle. La séance est scandée de phases d'institutionnalisation ayant pour contenu le même objet qu'une intervention ponctuelle antérieure. Ainsi, lorsqu'elle s'autorise l'émergence d'un savoir non prévu, celui-ci est très rapidement figé symboliquement pour toute la classe. Cette procédure interroge alors la fonction même de l'institutionnalisation pour D.

\section{L'ostension des savoirs chez D et E}

On présente ici un tableau dans lequel on dénombre les différentes procédures ostensives utilisées par les enseignantes pour chaque mise en jeu d'un savoir.

\begin{tabular}{|c|c|c|c|}
\hline & & $\begin{array}{c}\text { Enseignante } \\
\text { professionnellement } \\
\text { débutante (D) }\end{array}$ & $\begin{array}{c}\text { Enseignante } \\
\text { professionnellement } \\
\text { expérimentée (E) }\end{array}$ \\
\hline \multirow{4}{*}{$\begin{array}{l}\vec{D} \\
\tilde{n} \\
D \\
2 \\
0 \\
0 \\
0 \\
0 \\
0 \\
0 \\
0 \\
0 \\
0 \\
0 \\
0 \\
0\end{array}$} & $\begin{array}{l}\text { Nombre total } \\
\text { de mises en jeu } \\
\text { d'un savoir }\end{array}$ & 29 & 37 \\
\hline & Explications & 15 & 27 \\
\hline & Manipulations & 0 & 0 \\
\hline & Démonstrations & 16 & 11 \\
\hline \multirow{4}{*}{ 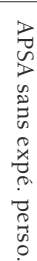 } & $\begin{array}{l}\text { Nombre total } \\
\text { de mises en jeu } \\
\text { d'un savoir }\end{array}$ & 13 & 10 \\
\hline & Explications & 13 & 10 \\
\hline & Manipulations & 3 & 3 \\
\hline & Démonstrations & 1 & 1 \\
\hline
\end{tabular}

Tableau 5: Récurrence des procédures ostensives chez D et E

Ce tableau met en avant une certaine similarité des enseignements de $\mathrm{D}$ et $\mathrm{E}$ vis-à-vis de l'ostension des savoirs. En effet, l'explication discursive constitue, d'une manière générale, le principal moyen ostensif utilisé par les enseignantes pour proposer des solutions aux élèves et répondre ainsi à leurs obstacles. À l'inverse, la manipulation reste relativement peu utilisée. Ensuite, dans les enseignements sans expérience personnelle, donc en lutte, les démonstrations sont quasiment absentes par manque d'expérience personnelle alors qu'elles sont récurrentes lorsque 
les enseignantes ont une expérience personnelle dans l'APSA enseignée. On peut toutefois remarquer que l'utilisation de la démonstration est extrapolée chez $\mathrm{D}$, en danse, puisque dans plus de la moitié des cas où elle met en jeu un savoir, elle le fait par l'intermédiaire de son corps. On peut enfin remarquer que le nombre total de mises en jeu de savoirs et surtout d'explications est prépondérant chez E.

Ainsi, d'une manière synthétique on peut relever que dans les APSA avec expérience personnelle, les enseignantes mettent en jeu davantage de savoirs et le font beaucoup plus par l'intermédiaire de démonstrations. Mais, il existe toutefois une tendance chez notre enseignante débutante à valoriser encore plus la démonstration lorsqu'elle connaît l'activité alors que notre enseignante expérimentée s'attache plus à y présenter les savoirs de manière discursive. Ces constats nous interrogent sur le statut de l'explication et de la démonstration dans la transmission ostensive des savoirs chez D et E.

\section{Interprétations : expérience personnelle et professionnelle et singularité du sujet enseignant dans le didactique}

Au moment d'interpréter les résultats que nous venons de présenter, nous devons rappeler notre objet de recherche. Il s'agit de rendre compte de l'importance que revêt, pour un enseignant d'EPS, le rapport qu'il entretient avec l'APSA qu'il enseigne. Nous avons fait l'hypothèse, étayée par une observation de la formation initiale et continue des enseignants d'EPS et par des travaux antérieurs (Leinhardt, Smith, 1985; Soler, 1994; Durand, 1997) qu'une différence très nette apparaît, pour le même enseignant et pour la même classe, entre l'enseignement d'une APSA qu'il connaît, qu'il a pratiquée, ou même dont il est spécialiste, et une APSA qu'il découvre et qu'il doit toutefois enseigner, du fait de la programmation de ce cycle dans son collège. Nous rappelons que pour mettre en évidence ce processus, nous avons observé deux enseignantes très différentes, puisque l'une est « expérimentée » et l'autre « débutante », faisant l'hypothèse que cette différence de statut pourrait nous donner des réponses à la question posée. Elles ont toutefois en commun le fait d'avoir à enseigner, pour des établissements et des classes comparables, deux APSA qui répondent à cette distinction. Ainsi, le lecteur ne sera pas surpris par cette proximité recherchée à des fins de comparaison, malgré leur différence d'ancienneté.

On se propose alors maintenant d'interpréter les résultats obtenus au regard de nos cadres d'analyse en didactique clinique, confrontés au discours des enseignantes dans les entretiens d'après-coup.

\section{Le rapport à la contingence}

Les enseignantes observées, comme tout professeur, se confrontent dans leur activité professionnelle à la contingence du processus de transmission des savoirs, elle-même déterminée par la contingence de l'activité d'apprentissage des élèves confrontés aux situations qui leur sont dévoluées. Une fois dans la classe, l'enseignant sait que les élèves mettront en œuvre des stratégies singulières, parfois inefficaces, pour répondre aux problèmes qui leur sont posés et que de nouveaux problèmes imprévus émergeront de la complexité de leur activité motrice adaptative. Il sait donc que son projet didactique ne s'accomplira pas sans détour, sans perte de contrôle potentielle. Il peut alors plus ou moins supporter cette perte de maîtrise, il peut la redouter, l'accepter, voire en jouir, dans la mesure où il sait lui-même y répondre. L'attitude de chacun face à cette part de non maîtrise dans la classe constitue ainsi son rapport singulier à la contingence.

Il nous semble alors que les résultats présentés révèlent des stratégies spécifiques pour gérer cette contingence qui, lorsqu'on les confronte au discours après-coup des enseignantes, révèlent des rapports singuliers à la contingence selon leurs expériences personnelles et professionnelles.

En effet, on peut observer au travers du tableau 3, plusieurs démarches d'enseignement révélant différentes stratégies pour gérer la contingence du processus didactique. Ainsi, l'enseignement de lutte mené par E semble montrer, en ne s'autorisant presque aucun écart par rapport à ses prévisions, une stratégie simple pour gérer la contingence. En ne répondant aux problèmes des élèves qu'à partir des savoirs qu'elle avait déjà prévu d'enseigner, E parvient à garder le contrôle didactique de la classe et à effacer ainsi une grande partie de la contingence du processus de 
transmission. Son discours après-coup montre alors la nécessité de cette stratégie, celle de se sécuriser visà-vis de l'imprévu: «En lutte, je mettais plusieurs situations sur le papier, pour moi, pour être sûre qu'il ne me manquerait pas encore du temps et que du coup, je doive improviser. Car je suis incapable de le faire, improviser ", "Je n'arrive pas à sentir moi-même l'essence pour arriver à les y amener. Donc, pour l'instant je me sécurisais». En revanche, la forme prise par les enseignements de danse et de badminton, dans lesquels les enseignantes font émerger de nombreux savoirs qu'elles n'avaient pas prévu d'enseigner, révèle un rapport bien différent à la contingence. Celle-ci n'est pas crainte lorsque les enseignantes ont une expérience personnelle dans l'APSA, contrairement aux enseignements de lutte. $\mathrm{D}$ : " je me sentais beaucoup plus utile en danse qu'en lutte ", "en lutte, j'avais l'impression de papillonner sur le tatami, sans rien donner de vraiment constructif, parce que je savais pas quoi leur dire ", "au niveau des contenus, un peu léger quand même ". E: "je ne possède pas l'activité, c'est la même, mais comme je ne la sens pas, j'ai du mal à la faire passer ", "C'est ça qui m'a le plus gênée, la méconnaissance ". C'est donc la méconnaissance, le manque d'expérience dans l'APSA, qui contraint les enseignantes à observer la contingence, en tentant de la gérer tant bien que mal, " gênées » d'être peu « utiles » à ne pouvoir que proposer des réponses identiques à chaque élève.

Toutefois, l'enseignement de lutte mené par D révèle une autre stratégie de gestion de la contingence. En effet, si D répète son sentiment d'inutilité en lutte, elle s'autorise quand même plusieurs fois, contrairement à E, à intervenir auprès des élèves à partir de savoirs qu'elle n'avait pas prévu d'enseigner. Sa planification ne semble pas être une voie de gestion de la contingence. Elle ne prétend pas d'ailleurs être incapable d'improviser. En revanche, la récurrence des phases d'institutionnalisation en continuité avec ses interventions ponctuelles, montre sa nécessité de figer toute émergence de savoir imprévu. Si la contingence est supportée, du fait qu'elle tente de s'y aventurer, elle le fait avec précaution, en s'appuyant sur une officialisation des nouveaux savoirs mis en jeu. «Ça fait un peu, pour pouvoir se dire... j'ai trouvé quelque chose à leur dire, alors autant en parler à tout le monde, au moins ça sera posé ". En posant le nouveau savoir, elle peut s'y appuyer et ainsi, ne pas rester dans sa simple planification tout en conservant une certaine maîtrise didactique de la classe.
Il semble donc que, dans leurs enseignements de lutte, D et E tentent, au travers de deux stratégies distinctes, de gérer la contingence en s'attachant à répondre à la diversité des obstacles des élèves à partir de solutions figées a priori ou in situ. Ces savoirs, qu'ils soient planifiés ou officialisés dans la classe, leur permettent ainsi de conserver une part de responsabilité dans la transmission des savoirs, sans avoir à s'aventurer trop loin dans l'imprévu, chose qui leur est impossible d'envisager et qui pourrait les placer dans une position ambiguë. Il semble ainsi que, malgré les vingt-cinq ans d'ancienneté qui les distinguent, le manque d'expérience personnelle de $\mathrm{D}$ et $\mathrm{E}$ les place dans un rapport similaire à la contingence, ce qui est une découverte pour nous, chercheurs en didactique de l'EPS. En revanche, l'expérience personnelle sur laquelle elles peuvent s'appuyer dans les enseignements de danse et de badminton, leur permet d'appréhender l'imprévu sans craindre de s'y perdre et donc sans avoir à le circonscrire à partir d'une planification ou d'un recours intensif à institutionnalisation. Toutefois, ces enseignements se distinguent à d'autres niveaux. Le discours dans « l'après-coup » de $\mathrm{D}$ et E révèle les causes de cette spécification.

\section{Le sujet supposé savoir}

La gêne et l'inutilité sont les sentiments éprouvés par ces enseignantes dans leurs enseignements de lutte. Si ce ressenti les amène à gérer le processus de transmission d'une manière spécifique, il semble que leur méconnaissance les place toutes les deux, à des degrés différents, dans une position contradictoire avec celle qu'elles sont censées détenir dans la classe. Or cette contradiction ne résonne pas comme un simple constat réflexif, mais plutôt comme un véritable problème à gérer en permanence. Car leur légitimité professionnelle réside principalement dans le savoir qu'elles sont censées détenir, pour pouvoir l'enseigner. Le manque de savoir retentit comme un manque de légitimité parfois difficile à assumer.

Ainsi, le discours après-coup des enseignantes à propos de l'ostension des savoirs met en avant la nécessité, largement extrapolée chez D, de rechercher et de conserver cette légitimité, c'està-dire d'assumer leur position symbolique dans la classe, celle de sujet supposé savoir. En effet, le 
recours intensif à la démonstration en badminton, et encore davantage en danse, ainsi que sa quasi absence en lutte, révèle cette nécessité. D introduit alors cette difficulté ainsi: " des fois tu ne maitrises pas du tout et le problème c'est que ça, ils le savent, ils le ressentent ». C'est alors la crainte d'être décelée dans l'usurpation de sa position symbolique qui la conduit à enseigner ainsi : "tu ne montres pas, parce que t'es flag (pris en flagrant délit) ». La forme prise par son processus de transmission est ainsi particulièrement déterminée par sa tentative permanente de ne pas être prise en flagrant délit de ne pas savoir. Si cette crainte est moins présente chez E, elle semble tout de même mal vivre cette impossibilité de démontrer: "je l'ai senti moi aussi qu'il aurait fallu que je leur montre ce qu'il fallait apprendre ", "par contre en lutte je ne l'aurai pas fait du tout... et ça je l'ai mal vécu». Nous verrons dans la dernière partie de nos interprétations que le «mal vécu » de E ne semble pas être autant déterminé que chez $\mathrm{D}$ par la nécessité d'assumer sa position symbolique. En effet, on peut encore davantage identifier cette nécessité dans l'enseignement de danse mené par $\mathrm{D}$. La récurrence de ses interventions et des démonstrations pour les appuyer ne semble pas renvoyer simplement à une stratégie didactique: "il y a un plaisir à asseoir ton autorité en montrant que tu maîtrises ", "la démonstration, c'est aussi leur en mettre plein la vue », "de temps en temps les calmer avec une bonne démonstration c'est pas plus mal».

Si les formes prises par les enseignements de lutte et ceux de danse et de badminton semblent être contrastées de la même manière et montrent le poids fondamental de l'expérience personnelle, il nous semble que l'extrapolation chez $\mathrm{D}$ de la nécessité de se légitimer met en avant le poids de l'expérience professionnelle. En effet, de par sa nouveauté dans la profession et donc sa nécessité de se forger une légitimité professionnelle, D semble en permanence rechercher, au-delà de ses préoccupations didactiques réfléchies, à endosser son statut de sujet supposé savoir.

Cependant, plusieurs aspects du processus de transmission des savoirs (l'institutionnalisation précoce de E en badminton, son mal-être en lutte et encore une fois l'interventionnisme de D en danse) nous semblent être déterminés par d'autres causes.
L'impossible à supporter

Au cours de nos travaux antérieurs sur l'analyse des pratiques d'enseignement in situ, nous avions observé certaines réactions incontrôlées de la part des enseignants observés. Ces réactions faisaient référence à des situations bien précises et relatives à leur propre expérience d'enseignant (Carnus, 2002) ou de pratiquant de judo (Loizon, 2004), comme la peur d'un accident, pour les élèves. Les entretiens d'aprèscoup nous avaient permis d'attribuer aux causes de ces réactions le statut d'impossible à supporter, en référence à la catégorie du Réel développée par Lacan. Il semble que les enseignements observés ici contiennent de telles réactions. Si la difficulté de D à supporter son illégitimité didactique en lutte en est un exemple, on peut en identifier plusieurs autres « impossibles à supporter » chez D comme chez E

En effet, comme on l'a montré, D, en danse, ne craint pas la contingence. Bien au contraire, dans un flot d'interventions incessant, elle s'engage à répondre à tous les problèmes spécifiques des élèves, qu'ils soient prévus ou non, qu'ils semblent bloquer leurs apprentissages ou pas. Son discours dans l'après-coup semble alors montrer que cet interventionnisme n'est pas simplement une manière d'affirmer sa position symbolique. Lorsqu'on l'interroge sur son ressenti vis-à-vis des élèves en danse, elle nous répond: « des fois ça ne ressemble plus à de la danse et ça, ça m'embête ", "j'avais peur... de rester dans les stéréotypes modern jazz... chorégraphie Jennifer ", "ça j’aime pas ", "ça, ça ne me plaît pas ". Elle semble ne pas pouvoir supporter l'activité adaptative maladroite des élèves qui s'engagent dans une pratique qui ne ressemble pas à celle qu'elle voudrait voir émerger. De son expérience clivée de danseuse classique et contemporaine, elle ne supporte pas le manque de rigueur esthétique ni les stéréotypes d'une danse médiatisée sans aucune intention expressive. Ainsi, lorsque les élèves pratiquent, elle considère qu'ils dénaturent l'activité telle qu'elle la conçoit et qu'elle l'a vécue et donc altèrent une part d'elle-même. Ainsi la récurrence de ses interventions semble être une voie pour retrouver l'essence de l'activité, qu'elle ne supporte pas voir s'effriter dans l'activité des élèves.

De son côté, E est confrontée à une autre forme d'impossible à supporter. Il renvoie au seul résultat que nous avons laissé de côté jusqu'à maintenant. En 
effet, qu'il s'agisse de la lutte ou du badminton, on a pu remarquer qu'elle donne toujours les solutions aux élèves dans des phases d'institutionnalisation avant même d'engager les processus de dévolution. Comme si elle ne supportait pas de voir les élèves s'engager dans une activité d'apprentissage coûteuse en énergie et en temps, elle s'attache toujours à leur donner la solution avant même qu'ils ne puissent la chercher: "moi, ça m'em... qu'ils passent à chercher deux heures des trucs que je sais", "j'essaie d'aller au plus vite à ce qui me paraît essentiel pour qu'ils progressent plus vite que s'ils avaient passé du temps à chercher les solutions ». Pour justifier sa démarche, E fait alors référence à sa propre expérience d'apprenante et à son ressenti déplaisant vis-à-vis de l'inefficacité des phases de recherche inhérentes à tout apprentissage. Elle donne alors l'exemple d'un stage de voile qu'elle a effectué en formation continue: "c'est complètement (idiot) de galérer toute une journée pour ne pas savoir pourquoi on était à l'eau ", "ils auraient pu expliquer, en deux minutes, on aurait pigé le truc". Puis, revenant sur ses propres difficultés scolaires, « je réussissais moyennement et cela me demandait beaucoup de boulot ", elle explicite cet impossible à supporter: "je n'aime pas que les gens soient en difficulté et n'arrivent pas à s'en sortir ». Il semble ainsi qu'il lui est particulièrement difficile de supporter la position instable, et parfois angoissante, dans laquelle elle pourrait placer les élèves en les laissant prendre la responsabilité de chercher les solutions aux problèmes dévoluées. Leur donner initialement et collectivement la solution avant même qu'ils ne la cherchent lui permet d'esquiver cet insupportable. Son «mal-vécu » en lutte semble ainsi pouvoir être attribué à son impossibilité de donner pertinemment les solutions aux élèves.

\section{Conclusion}

Notre intention était d'identifier l'impact des expériences personnelles dans les APSA et professionnelle dans l'enseignement scolaire du sujet enseignant sur l'enseignement effectif de ces dernières.

À partir de l'analyse croisée de ces deux études de cas, on peut s'apercevoir que les ressorts du fonctionnement singulier du processus de transmission des savoirs de chaque enseignante peuvent gagner en intelligibilité, lorsque le chercheur prend en compte les enjeux subjectifs qui organisent l'activité $\mathrm{du}$ professeur d'EPS. Lorsque ce dernier enseigne, il doit aussi légitimer sa position symbolique, maîtriser l'incertitude dans laquelle il est plongé, ou encore, accepter de voir une partie de son savoir se dénaturer dans l'activité des élèves.

Les résultats que nous venons de présenter ne sont évidemment pas généralisables. Nous avons procédé à deux études de cas contextualisées, dans le seul but d'observer et de rendre compte de l'effet, sur les pratiques d'enseignement de l'EPS, de la connaissance personnelle et corporelle d'une APSA. Pour y parvenir, nous avons utilisé une « posture » clinique, avec une méthodologie d'étude de cas, un recueil de données sous forme d'enregistrements vidéos et d'entretiens, et surtout en croisant des références théoriques de la didactique et de la clinique lacanienne.

Les outils d'analyse que nous avons utilisés nous ont été fournis par l'emprunt à ces deux champs scientifiques, mais aussi aux travaux qui se réclament de la didactique clinique. Nous n'avons que poursuivi cette voie, en confortant certains éléments d'analyse, qui nous semblent pertinents (mais peu utilisés) dans l'analyse des pratiques. En parallèle des résultats qui sont relatifs à ces observations ciblées, nous souhaitons que soient retenus et développés les cadres d'analyse qui ont été utilisés ici, comme ceux de:

- « rapport à la contingence », qui rend compte de la façon dont les enseignants intègrent l'incertitude dans laquelle ils sont plongés dès lors qu'ils enseignent, celle de ne pas savoir quand, comment et par quelles voies l'élève apprend,

- « sujet supposé savoir», qui situe l'enseignant dans sa fonction symbolique jamais totalement assurée de la transmission de savoirs,

- « impossible à supporter », qui renseigne sur ce qui pourrait constituer le symptôme de l'enseignant, soit le grain de sable qui grippe la machine, souvent à son insu.

L'articulation systémique entre ces trois cadres permet de proposer une première modélisation du sujet didactique, situé au croisement de contraintes didactiques et internes. On peut la décrire de la 
manière suivante. Toute situation d'enseignement est contingente. Ainsi, le sujet enseignant, comme chacun face à la contingence, se doit d'apporter sa part de maîtrise dans cette incertitude. Or, de par son statut institutionnel, il a aussi une position symbolique à assumer, celle de sujet supposé savoir, qui le place dans une posture de maîtrise supposée. La maîtrise absolue, si elle est une des principale finalité de toute activité, ne s'actualise dans la classe que comme un processus de recherche de cette maîtrise, toujours impossible à atteindre. Ainsi, divisé entre une position symbolique supposée et une position subjective réelle, l'enseignant se confronte à des formes d'impossible à supporter qui orientent son activité didactique.

Nous avons conscience de creuser une voie difficile, à la croisée de deux champs théoriques différents, mais qui nous semble toutefois pertinente et heuristique pour l'analyse plurielle des pratiques d'enseignement. La tentative de construire des outils théoriques ayant une certaine portée générique nous semble aussi révéler la capacité de la didactique clinique à contribuer au développement de la didactique comparée. 


\section{RÉFÉRENCES}

Altet, M. (2002). L'analyse plurielle de la pratique enseignante: une démarche de recherche. Revue Française de Pédagogie, 138.

Artigue, M. (1990). Ingénierie didactique. Recherches en didactique des mathématiques, 9.

Beillerot, J. (1989). Savoir et rapport au savoir. Élaborations théoriques et cliniques. Paris: Éditions universitaires.

Blanchard-Laville, C. (2001). Les enseignants entre plaisir et souffrance. Paris: PUF.

Blanchard-Laville, C., Fablet, D. (2000). L'analyse des pratiques professionnelles. Paris: L'Harmattan.

Brousseau, G. (1986). Théorisation des phénomènes d'enseignement des mathématiques. Thèse d'État de l'Université Bordeaux 1.

Brousseau, G. (1998). Théorie des situations didactiques. Grenoble: La Pensée Sauvage.

Bru, M. (2004). Les pratiques enseignantes comme objet de recherche. In J.-F. Marcel (Dir.), Pratiques enseignante hors de la classe (p.. 281-299). Paris: L'Harmattan.

Brun, J. (1996). Évolution des rapports entre la psychologie du développement cognitif et la didactique des mathématiques. In J. Brun (Ed.), Didactique des mathématiques. Paris: Delachaux et Niestlé.

Buznic-Bourgeacq, P., (2005). L'enseignement: une conversion de l'expérience de l'enseignant. Colloque recherche et formation, former des enseignants-professionnels. Nantes, Fév. 2005.

Charlot, B. (1997). Du rapport au savoir. Éléments pour une théorie. Paris: Anthropos.

Chevallard, Y. (1989). Le concept de rapport au savoir : rapport personnel, rapport institutionnel, rapport officiel. Actes du séminaire de didactique. Grenoble: IMAG.

Chevallard, Y. (1991). La transposition didactique. Grenoble: La Pensée Sauvage.

Carnus, M.-F. (2002). Une approche didactique du processus décisionnel de l'enseignant d'EPS en situation didactique. In P. Venturini, C. Amade-Escot, A. Terrisse, Études des pratiques effectives: l'approche des didactiques (p.. 83-92). Grenoble. La Pensée Sauvage.

Durand, M. (1997). Teaching task and teaching strategies. In Vanden, Auweele, Bekker, Biddle, Durand, Seiler (Eds.), Psychology for Physical educator. Champaign: Human Kinetics

Giordan, A. (1996). Représentations et conceptions en didactique. In P. Clément, Regards croisés sur les STAPS. CRDP d'Alsace.

Lacan, J. (1966). Écrits. Paris: Le Seuil.

Leinhardt, G., Smith, D.A. (1985). Expertise in mathematics instruction: subject-matter knowledge. Journal of education psychology, $77 / 3$.

Lestel, G. (2005). Analyse didactique et clinique de la transmission des savoirs: une étude de cas en EPS. Mémoire de mastère 2 de l'Université Toulouse 2.

Loizon, D. (2004). Analyse des pratiques d'enseignement du judo: identification du savoir transmis à travers les variables didactiques utilisées par les enseignants en club et en EPS. Thèse de doctorat de l'Université Toulouse 3.

Martinand, J.-L. (1994). Pratiques de références, transposition didactique et savoirs professionnels en sciences et techniques. Les sciences de l'éducation pour l'ère nouvelle, 1/2.

Mercier, A. (1999). L'espace-temps didactique, étude du didactique, en sciences de l'éducation. Note de synthèse pour l'HDR de l'Université d'Aix-Marseille.

Perrin-Glorian, M.-J. (1994). Théorie des situations didactiques: naissance, développement, perspectives. In Artigue, Gras, Laborde, Tavignot, 20 ans de didactique des mathématiques en France. Grenoble: La pensée sauvage. 
Salin, M.-H. (2002). Les pratiques ostensives d'un enseignement des mathématiques comme objet d'analyse du travail du professeur. In P. Venturini, C. Amade-Escot, A. Terrisse (dir.), Étude des pratiques effectives: l'approche des didactiques. Grenoble: La Pensée Sauvage.

Sensevy, G., Mercier, A., Schubauer-Léoni, M.-L., (2000). Vers un modèle de l'action didactique du professeur à propos de la course à 20. Recherches en didactique des mathématiques, 20/3.

Sensevy, G. (2002). Des catégories pour l'analyse comparée de l'action du professeur: un essai de mise à l'épreuve. In P. Venturini, C. Amade-Escot, A. Terrisse (dir.), Étude des pratiques effectives: l'approche des didactiques. Grenoble: La Pensée Sauvage.

Soler, A. (1994). Contribution à l'étude des connaissances du contenu chez les enseignants d'EPS. Mémoire de DEA de l'Université de Montpellier 1.

Terrisse, A. (1994). La question du savoir dans la didactique des APS: essai de formalisation. Note de synthèse pour l'HDR de l'Université Toulouse 3.

Terrisse, A. (2000). Épistémologie de la recherche clinique en sports de combat. In A. Terrisse (dir.), Recherches en sports de combat et en arts martiaux: état des lieux. Paris: Ed. Revue EPS.

Terrisse, A. (2001). Didactique des disciplines: Les références au savoir. Bruxelles: De Boeck Université.

Tochon, F. (1996). Grammaire de l'expérience et savoirs-objets: le savoir focal dans la construction de nouveaux modèles de formation. In J.-M. Barbier, Savoirs théoriques et savoirs d'action. Paris: PUF.

Van Der Maren, J.-M. (1995). Méthodes de recherche pour l'éducation. Bruxelles: De Boeck Université.

Venturini, P., P., Amade-Escot, C., Terrisse, A. (2002). Étude des pratiques effectives: l'approche des didactiques. Grenoble: La Pensée Sauvage

Verret, M. (1975). Le temps des études. Lille: Librairie Champion. 